\title{
THE DYNAMIC ERGODIC DIVERTOR (DED)
}

\author{
K.H. Finken \\ Institut für Plasmaphysik, Forschungszentrum Jülich, Euratom Association \\ Trilateral Euregio Cluster, 52425 Jülich,Germany \\ TEL. +49 246161 5646; Fax +49 2461615452
}

\section{ABSTRACT}

The Dynamic Ergodic Divertor (DED) has recently been taken into operation on the TEXTOR tokamak. The main aim in this context is the study of its divertor properties. After an introduction into the theory of ergodization, the design of the DED is presented. The Chirikov parameter describing the level of ergodization reaches a level of up to four if the perturbation current is applied in an optimized way. Finally the rich physics options of the DED are discussed.

\section{INTRODUCTION}

During the last two decades, considerable progress has been made in improving the plasma confinement. The sufficiently low error margin from the different devices makes it now possible to extrapolate the confinement data to a reactor scenario such it can be expected that the plasma of the proposed ITER experiment will most likely ignite. The essential ingredient for a good confinement is the existence of magnetic flux surfaces which form - typically eccentric - onion shell like structures inside the fusion devices. Magnetic field lines stay always on "their" magnetic surface and these surfaces also form isobars.

A key element for obtaining the good plasma confinement quality is the poloidal divertor which allows for a relatively easy access of the high confinement mode $(\mathrm{H}-$ mode) of a tokamak. The H-mode operation is considered the standard scenario for ITER. In the H-mode, a barrier is formed at the plasma edge which - together with an observed profile stiffness -leads to the overall improvement of the confinement. However, the confinement in the plasma edge tends to be so good, that new edge instabilities develop, the so called Edge Localized Modes, ELMs. The ELMs affect only the plasma pedestal and are therefore at a first glance not harmful with respect of the confinement. However, the ELMs show a sharp rise in the characteristics of ejected energy and particle efflux and a short duration. At present day tokamaks, ELMs are disturbing but not harmful. For ITER, however, the power to the walls is so high that it may determine the lifetime of the device. This is one of the reasons why ergodicity and ergodic divertors attract recently high attention to the fusion community ${ }^{1}$.

Ergodization of magnetic field lines is used in this context in contrast to "good magnetic surfaces" where a magnetic field line remains on one surface; ergodic magnetic field lines span up a whole volume. In particular, ergodicity means that any magnetic field line comes infinitely close to any point in an ergodized volume. Ergodization results from perturbations which are resonant to specific values of the safety factor $q$. When using an external perturbation, one can select whether one ergodizes preferentially the inner surfaces or surfaces closer to the edge. In the first case, the "woven" magnetic field lines form an internal ergodic layer which is typically characterized by an enhanced radial transport of particles and energy. In the second case one generates in addition to the ergodic field lines those which leave the plasma and intersect the walls. These field lines will carry enhanced fluxes of particles and energy to the wall and will lead to areas of enhanced plasma-wall interaction. In this way, an open chaotic system is formed.

The open magnetic field lines, i.e. those which intersect the wall twice, form the so called laminar zone. This zone is equivalent to the scrape-off layer (SOL) of a poloidal divertor; however, in contrast to the conventional SOL, the connection lengths of the magnetic field lines is not uniform but consists of magnetic flux bundles with multiples of a poloidal turn. The investigation of structure of the laminar zone, of the ergodic zone and the consequences for the transport are of particular interest $\mathrm{t}^{2,3}$.

The topics of research on the DED in Juelich are:

- the investigation of the properties of a helical divertor,

- the analysis of the transport in the flux tubes of the plasma edge,

- plasma shielding,

- the distribution of the average power flux to the walls,

- study of the edge electric field and modifications of the plasma rotation,

- the reduction and mitigation of ELMs,

- enhancing of the operational limits such as density limit ${ }^{4}$, - effects of the DED on the plasma confinement, 
- modification of the transport inside the plasma,

- the excitation of MHD-modes by the DED-field,

- a reduction of excited tearing modes by the DED.

The DED has been designed such that it can operate in different modes such as static or dynamic, in a fine perturbation mode $(m / n=12 / 4)$ and in a coarse mode $(m / n=$ $3 / 1)$. Different scenarios may optimum for one condition and therefore this specific point can be investigated only in one specific mode of operation.

In the following, a short introduction into the background for the ergodization is provided, followed by a description of the proposed DED experiment, then the magnetic field line structure is discussed and some experimental results, in particular those with the DED divertor aspects will be presented; finally the dynamic option is highlighted.

\section{THE DED-ARRANGEMENT}

The coil arrangement of the $\mathrm{DED}^{2,5,6}$ is shown in Fig. 1. It consists of a quadruple set of four helical conductors, installed on the inboard side of the TEXTOR vessel and aligned parallel to the magnetic field lines (for $\beta_{\text {pol }} \approx 1$ ) at the nearby $q=3$ surface. Taking into account the available space, the technical constraints (such as current density, skin effect, heat capacity, cooling aspects, etc.) and the physics requirements, an $m=12, n=4$ perturbation field structure has been selected. This can be achieved by using coils which cover about $30 \%$ of the inboard vessel surface on the high field side and which will be energized by a 4-phase current (up to $15 \mathrm{kA}$ ) at selected frequencies (DC, $90 \mathrm{~Hz}, 1 \mathrm{kHz}$ and a band of $1 \mathrm{kHz}$ to $10 \mathrm{kHz}$ ). These frequencies correspond to a rotation of the perturbation field around the torus similar to the propagating field of an $\mathrm{AC}$ motor; the phase velocities projected on the poloidal coordinate of $\mathrm{v}=\omega \mathrm{r} / \mathrm{m}=65 \mathrm{~m} / \mathrm{s}$ at $90 \mathrm{~Hz}$, and $7225 \mathrm{~m} / \mathrm{s}$ at $10 \mathrm{kHz}$ respectively; for the $m=3, n=1$ base mode, the velocity is four times higher..

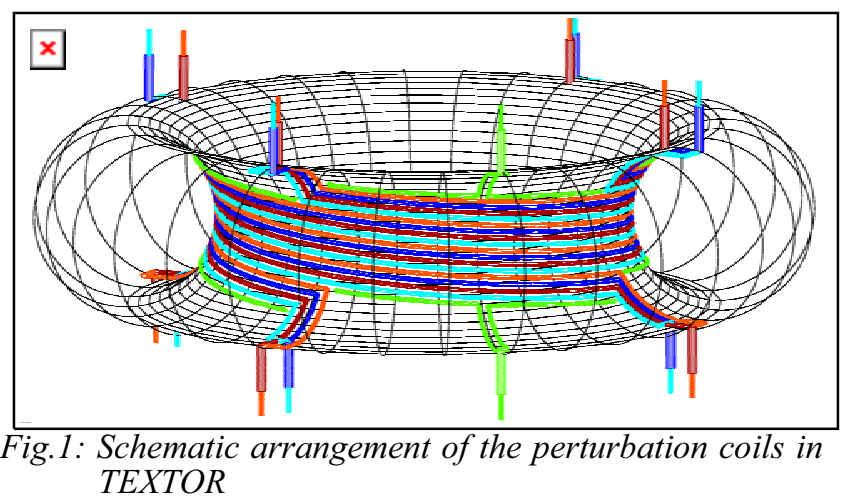

The coils are bundled such that the outlets are at 4 toroidal locations, 4 on top of TEXTOR and 4 at the bottom.
This grouping of the coils is technically favorable, but it requires the installation of a pair of compensation coils which are indicated in green color. The coils are covered by ceramic tiles and by $2 \mathrm{D}$ shaped graphite tiles forming a smooth toroidal surface, the divertor target plate.

\section{THE ERGODIC STRUCTURE GENERATED BY THE DED}

The ergodic structure resulting from this perturbation is often shown in the so-called Poincaré plot. In this plot a characteristic plane has to be defined which for a tokamak is a poloidal cut of the torus with the coordinates $r$ for the minor radius and $\Theta$ for the poloidal angle. In this plane starting points for magnetic field line tracing are selected and marked by a dot. The field lines are traced many times (typically several 1000 times) around the torus and every time the location of the intersection of the field line and reference plane is marked by a dot (puncture plot). If the perturbation field is not applied, the puncture sequence will remain on a flux surface - in the Poincaré plot thus forming a closed curve.

If the starting point is on a (preferentially low) rational q-number surface, the curve will degenerate to individual points - e.g. at the $q=3$ surface to three points per starting condition. With applied perturbation field, the pattern will be modified in a characteristic way. The big advantage of the Poincare representation is that the 3-D chaos problem can be cast into a 2-D picture which is easily graphically represented. Two Poincaré plots for the TEXTOR DED are shown in Figs. 2 and 3. The figures are cut at the outer equator $(\Theta=0)$ and unfolded; the abscissa is the poloidal angle and the ordinate the radius. This representation has been selected to enhance the radial resolution with respect to the poloidal one. In Fig. 2 the perturbation current is relatively low, $30 \%$ of the maximum allowed value. The rectangle on the center top represents the location of the DED coils and the object on the top left is the pump limiter ALT-II.

The Poincaré plot shows all the structures expected in the sub-ergodic phase: At the $q=3,3.25$ and 3.5, the main islands are formed with mode numbers $m / n=12 / 4,13 / 4$ and 14/4 ( $m$ stands for the number of poloidal knots and $n$ for the one of toroidal ones; the resonance condition requires $q=m / n$ ). Towards the plasma core (bottom part of the figure) the flux surfaces are nearly unchanged resulting in well ordered puncture curves. In between the main islands, intact surfaces are clearly visible. Between the $q=3$ and $q=3.25$ surfaces, smaller island chains are found; these and sub-islands in the main islands result from second order perturbation theory. The structures are well described by the KAM (for Kolmogorov, Arnold and Mozer) theory which is treated in text books ${ }^{3-7}$. 
With increasing perturbation amplitude, the island width increases and the islands start to overlap. In particular the region between the $q=3$ and $3=3.25$ surfaces becomes ergodic, i.e. field lines from any starting point reach any other point in that area. Between the $q=3.25$ and the $q=3.5$ surfaces, a barrier surface stays intact at low perturbation levels preventing a "diffusion" of the field lines. With increasing perturbation strength, also the barrier disappears in the whole boundary and allows for an enhanced field line diffusion as shown in Fig. 3.

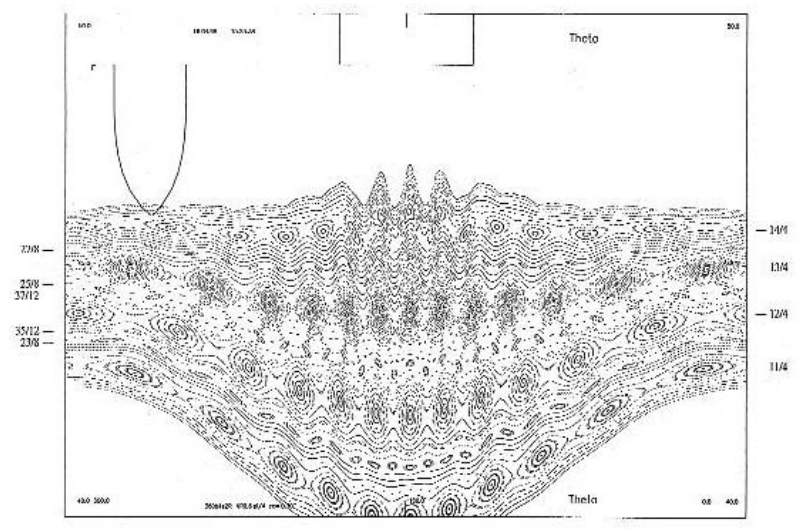

Fig.2: Poincaré plot for a low perturbation field $\left(I_{\text {pert }} / I_{\max }=0.3\right)$. As expected, magnetic islands develop at low rational q-values. The ordinate represents the radial direction (plasma side to the bottom) and the abzissa is the poloidal angle.

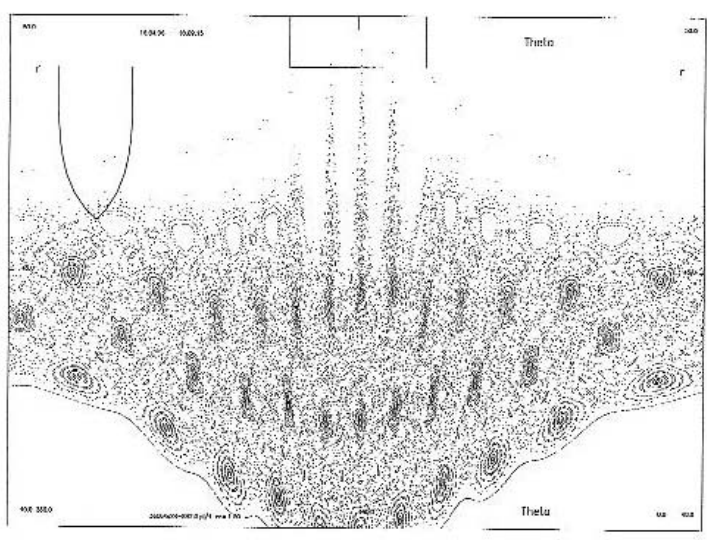

Fig. 3: Poincaré plot for the perturbation current condition $I_{\text {per }} / I_{\max }=1$. The whole outer plasma boundary is ergodized

\section{HAMILTON FORMALISM FOR MAGNETIC FIELD LINE TRACING}

One important method to describe the development of "weak" ergodization of magnetic field lines uses the Hamiltonian formalism. This method is applicable because dis- sipation does not occur ( $\operatorname{div} B=0)$ and the problem can be cast into a Hamiltonian form; therefore important laws as the conservation of the phase space can immediately be deduced.

The Hamiltonian form for the field line tracing can be applied if the magnetic field depends at most weakly on one coordinate. In a Tokamak or Stellarator this is e.g. the toroidal coordinate. For simplicity let us consider here a magnetic field with the main component in z-direction. The equation of motion for field line tracing is then written

$$
\text { as: } \begin{aligned}
\quad \frac{d x}{d s} \cong \frac{d x}{d z}=\frac{B_{x}}{B_{z}}=\frac{\partial a_{z}}{B_{z} \partial y}-\frac{\partial a_{y}}{B_{z} \partial z} \\
\frac{d y}{d s} \cong \frac{d y}{d z}=\frac{B_{y}}{B_{z}}=\frac{\partial a_{z}}{B_{z} \partial x}-\frac{\partial a_{x}}{B_{z} \partial z}
\end{aligned}
$$

By equating $\mathrm{t} \equiv \mathrm{z}, \mathrm{q} \equiv \mathrm{x}, \mathrm{p} \equiv \mathrm{y}, \mathrm{H} \equiv \mathrm{a}_{\mathrm{z}} / \mathrm{B}_{\mathrm{z}}$ and assuming that the derivatives with respect to $\mathrm{z}$ are of the order $\varepsilon$ only the equations of motion for the field line tracing become: $:^{7,8,9,10}$

$$
\begin{aligned}
& \frac{d q}{d t}=\frac{\partial H_{o}}{\partial p}+\varepsilon \cdot \frac{\partial f}{\partial t} \\
& \frac{d p}{d t}=-\frac{d H_{o}}{d q}+\varepsilon \cdot \frac{\partial g}{\partial t} \\
& \frac{\partial f}{\partial p}=\frac{\partial g}{\partial q}
\end{aligned}
$$

If $\varepsilon=0$, i.e. if the problem does not depend on $z$, this formalism describes a 1-dimensional motion of a particle. The system is integrable and the path curve is not subjected to any ergodization. A rather simple analogon is the motion of a pendulum ${ }^{11}$ which has been analyzed extensively with respect to the development of chaos and which is considered prototypical: The pendulum frequency depends on the amplitude of the motion; it spans a frequency band from its maximum at small amplitudes to zero frequency at the separatrix (which divides the oscillating motion $(\mathrm{H}<0)$ from the free rotation $(\mathrm{H}>0))$.

For $\varepsilon>0$, the Hamilton function depends on time and the problem has a dimension of 1.5. For the pendulum, this corresponds to the interaction with an external force. If this force has one Fourier component with one frequency in the band of the unperturbed motion, a resonance close to a specific amplitude is imposed and the motion has a maximum disturbance there. The resonant excursion cannot grow to infinity since otherwise the resonance condition is lost again; in the phase space, the resonance forms an "island".

If the Fourier spectrum of the perturbation has more than one frequency in the band of the unperturbed motion, several resonant islands are generated. The width of the island increases with the perturbation level; at a sufficiently high amplitude they start to overlap. From then on, the motion jumps irregularly from one island to another and in this band it becomes chaotic or ergodic. 
The formulae of the most important quantities in the ergodization theory are given below, namely for the island width $\Delta_{m n}$, the poloidal and toroidal mode numbers $m$ and $n$, the Chirikov parameter $\sigma$, the Kolmogorov length $L_{k}$, and the quasi linear diffusion coefficient $D_{Q}$. A magnetic island is generated (in first approximation) at the magnetic surface with the safety factor $q\left(r_{m n}\right)=m / n$ by an perturbation field which has a spatial Fourier component $b_{m n}$ at $r_{m n}$ :

$$
\Delta_{m n}=\sqrt{\frac{16 r b_{m n}}{m B_{t}} \cdot L_{s h}}
$$

here $B_{t}$ is the toroidal field strength and $\mathrm{L}_{\mathrm{sh}}$ is the shear length

$$
L_{s h}=\frac{q^{2} R_{0}}{r} \cdot 1 / \frac{d q}{d r}
$$

The Chirikov parameter characterizes the overlap of different islands and is a measure for the degree of ergodicity:

$$
\sigma(r)^{2}=\frac{8 r\left(b_{m n}+b_{m i n}\right)}{m\left|L_{s h}\right| B_{t}} \cdot\left(\frac{m q R}{r}\right)^{2}
$$

for $0 \leq \sigma \leq 1$, islands dominate the magnetic stucture while for $\sigma>1$ the magnetic field line pattern is ergodic. The Kolmogorov length characterizes the distance from where on initially neighboring field lines start the separate exponentially:

$$
L_{k}=2 \pi q R_{0} \cdot \sigma^{-4 / 3}
$$

The quasi linear field diffusion coefficient finally is

$$
D_{Q}=2 \pi q R_{0} \cdot\left(\frac{b_{m n}}{B_{t}}\right)^{2}
$$

particle diffusion coefficients are linked with $D_{Q}$ by a multiplication of a characteristic velocity for either ions or electrons.

\section{V.THE LAMINAR ZONE}

For the practical considerations, the plasma edge is best characterized by laminar plots ${ }^{12}$ and Poincaré plots; both plots are essential for the later analysis of the data in the rather complicated 3D structured plasma edge. As already mentioned, the laminar plot is characterized by the connection lengths of the magnetic field lines with the walls. We typically construct a laminar plot by choosing a poloidal cut of the plasma edge e.g. at the low field side as shown in Fig.4. In this example the poloidal axis spans from $-30^{\circ}<\Theta<30^{\circ}$ and the minor radius from $44 \mathrm{~cm}$ to $48 \mathrm{~cm}$. From each point of this area we start magnetic field line tracing in both directions until the magnetic field line intersects the wall. The total connection length is then decoded into the figure e.g. by coloring the points. The areas with the longest connection length are plotted dark (red) and the ones with a shorter connection length in lighter colors. The longest connection length we count to the laminar zone is 5 poloidal turns. The field lines with the longer connection lengths stem from the ergodic zone, the island region or even the confinement region. These areas have to be investigated in more detail with Poincaré plots.



Fig. 4: The laminar plot gives the connection lengths of the magnetic field lines in color coding; the right bar refers the colors to the lengths. The laminar zone corresponds to the scrape-off layer of a poloidal divertor, however, with more complicated connection lengths patterns.

Because of the symmetry of the DED configuration, we have 4 independent areas of the laminar plots which are image-symmetric. These are those plots where field lines have the same length to the two intersections in front of the DED; the plasma flows connected with these flux tubes form there a stagnation point. Two of these stagnation points are at the low field side, namely for those magnetic flux bundles with an odd number of poloidal turns between the intersection with the walls and two others for those of even connection lengths. In figure 4 the area "S" has a connection length of 3 poloidal turns and "S" represents the stagnation point of those flux tubes.

The simplest structure of the laminar zone is the one formed by the single turn field lines which correspond to the conventional scrape-off layer (the number of toroidal turns is q times the poloidal number). In contrast to the poloidal divertor case, these areas are no longer toroidally symmetric but show strong indentations. On laminar plots of the high field side, one can also recognize field lines with even shorter connection length, the private flux zone.

The light (blue) area represents field lines which intersect the divertor target plate after two poloidal turns. Be- 
cause the field lines move twice around the torus, they must appear twice in the figure. For this reason, the left and right "oval" structures have the same area and are interconnected by the field lines.

The area of three poloidal turns forms the sickleshaped pattern "S" of figure 4 . The field lines of this area must reappear twice and they form the trapezoid structures next to the oval structure. Figure 5 shows the development of the sickle-shaped magnetic flux tube along its path in one toroidal direction. The contour line of the area is mapped every $90^{\circ}$ and the numbers indicate the mapping step. The starting point is at subfigure " 0 " (left bottom). The shape of the is very little modified as long as one stays away from the DED coils (-1 .. -3). By the DED field, the flux tube is strongly deformed and elongated reflecting the trapezoidal figure $(-4 . .-6)$. Outside of the DED area, the flux tube experiences only the shearing of the equilibrium field (-7 .. -13). Then when entering again the DED zone, the flux tube is further deformed and deflected towards the divertor target plate where the mapping is stopped.

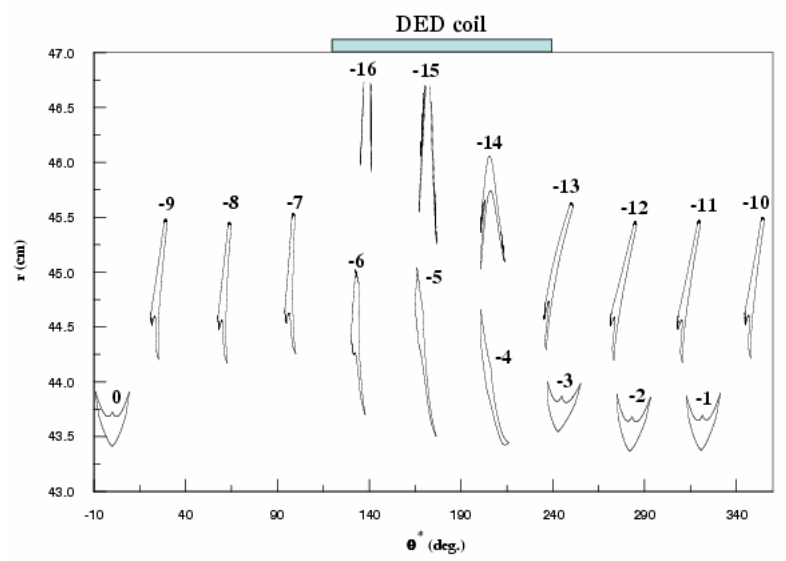

Fig. 5: Deformation of a magnetic flux tube obtained by field line mapping; the starting point is the stagnation point " 0 " and the end point \#-16 when the flux tube intersects the wall.

The higher order flux tubes become increasingly small and merge with the ergodic structure. However, an important ingredient is the set of flux tubes which interconnect the ergodic area with the walls. The flux tubes are rather thin and are called "fingers" or "tangles". Since these flux tubes come from a deeper zone of the plasma, the ergodic zone, it is expected they are effective in carrying heat and particles towards the walls. Part of the particles and power will diffuse thereby to neighboring field lines in the laminar zone such that the deposition pattern will be smeared out. Details of this redistribution will depend on the edge density and temperature. For understanding of the transport, several modeling codes ${ }^{13,14}$ of the area of both the ergodic zone and laminar zone have been performed.
The electrons in the plasma boundary are cooled by ionization and radiation; with ergodization, it is lowered in addition by the enhanced heat conductivity along the field lines and as a consequence, the ionization and excitation zones are broadened and shifted inward. Moreover, the impurity release due to energy dependent processes (e.g. sputtering) can be reduced significantly. Thus the combined effects of the increased electron density and decreased electron temperature at the plasma boundary in conjunction with the enhanced radial transport are responsible for the beneficial effects observed on devices with ergodic divertors ${ }^{15,16,17}$; from those the expected improved radiation efficiency of the impurities and the better impurity screening is an issue for TEXTOR experiments.

\section{EXPERIMENTAL RESULTS}

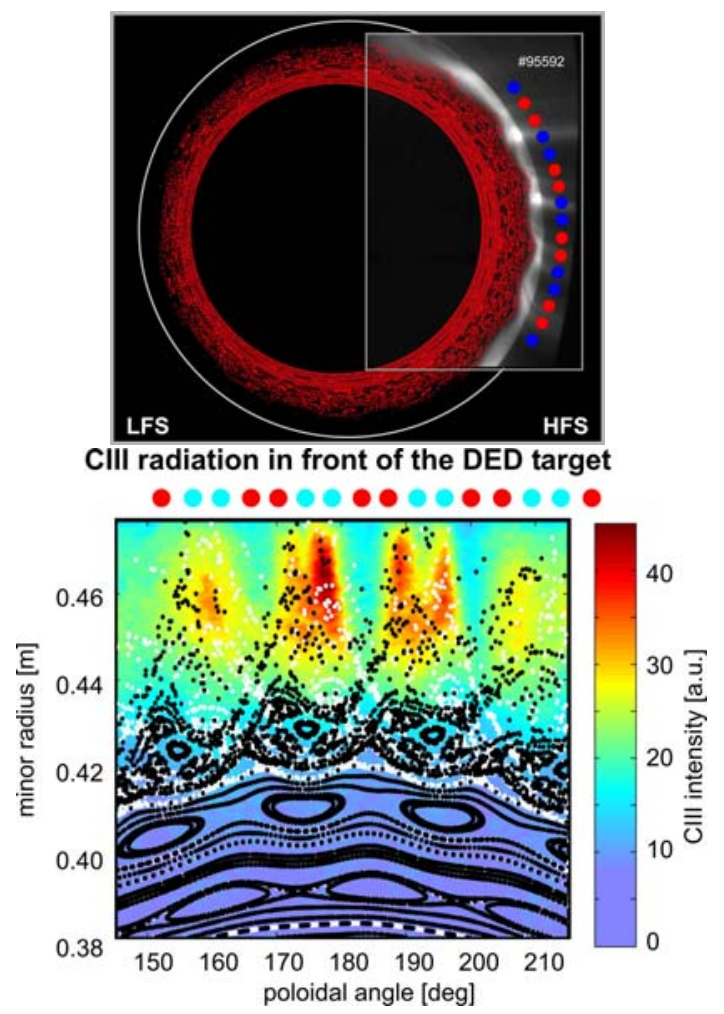

Fig. 6 top: Overview of the plasma edge structure of the DED-dominated plasma in the light of CIII emission. Superimposed are the location of the DED coils and the Poincaré plot of the magnetic field lines.

The bottom subfigure is rotated by $90^{\circ}$ and it shows the details of the "fingers".

An overview over the edge structure of the DED plasma is shown in Fig. 6. Plotted is the CIII line emission of carbon recycling at the divertor target plate. In addition the location of the DED coils is indicated by the dots representing the two directions of the electrical current. Overlaid to the picture is a Poincaré plot of the magnetic field 
lines in the edge. One sees that the light of the recycling particles follows nicely the structure imposed by the DEDfield.

The maximum interaction is - as expected - close to the location where the fingers touch the wall. One also sees that the radius of the ergodization zone amounts to $10 \%$ of the minor radius. The lower sub-figure shows the interaction zone in higher detail. The fingers are plotted either as black or as white dots depending on the direction of field line tracing. The lower figure is tilted by $90^{\circ}$ with respect to the upper one.

The slow rotation of the DED with few $\mathrm{Hz}$ only allows the visualization of 3D structures during local measurements. Because one can program only positive DED currents in this case, the rotation angle is limited to $1 / 4$ of the full phase. Fig 7 shows on the left side a laminar plot at the low field side and the right sub-figure gives the radially resolved electron pressure derived from a helium beam signal. The laminar plot has been evaluated for the position of the diagnostics. The yellow square in the left figure gives the range over which the DED-field moves during the rotation. The electron pressure variation in right subfigure agrees well with the expectation of the field line tracing: For the given phase of measurement the plasma boundary at the location of the diagnostic moves to the inner part of the plasma.

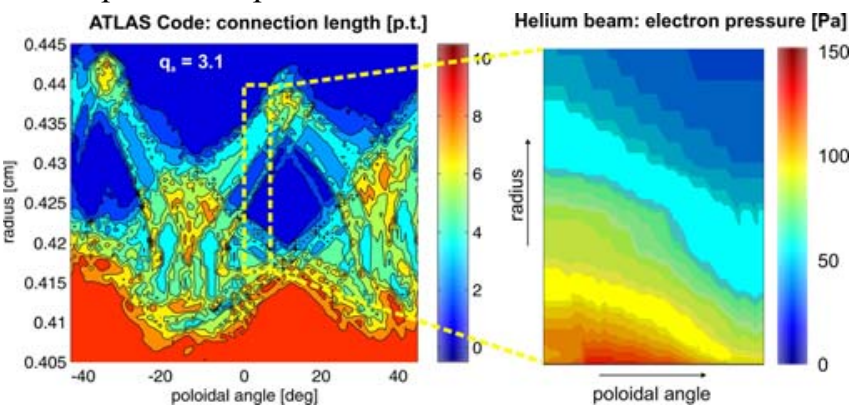

Fig. 7: By a slow rotation of the DED-field, the 3-D structure of the plasma boundary can be shown even for a measurement which records data along a radial line as shown here for the atomic helium beam diagnostic. The left subfigure gives a laminar plot while the right one is the actual measurement for the yellow square.

Another diagnostic technique is ECE imaging which provides a $2 \mathrm{D}$ picture of the electron temperature. Shown in Fig. 8 is again a laminar plot and the temperature distribution for the area of the yellow rectangle. One sees that the area of the fingers is hotter than that area which is strongly connected with the plasma wall. Again the temperature field agrees well with the expectations, even though the measurements are performed at the low field side, far away from the DED; the properties of the plasma in the magnetic flux tubes are strongly determined by the parallel transport.

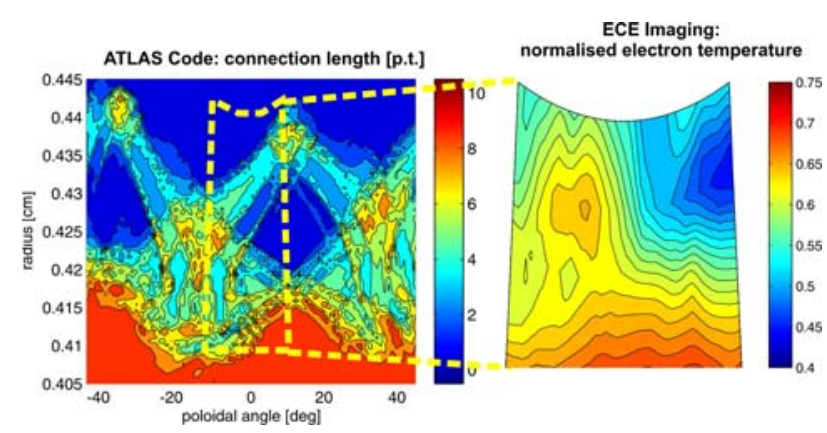

Fig. 8: ECE imaging provides directly a 2-D image of the electron temperature in the laminar zone as shown in the right subfigure. The left subfigure gives again the corresponding laminar plot similar as in the previous figure.

The heat flux pattern at the divertor target plate is dominated by the deflection of the magnetic field lines in front of the DED coils ${ }^{18}$. On therefore obtains a helical pattern as shown in Fig. 9; again a good agreement is found between the predicted laminar and footprint plots and the measured ones. The DED resembles a poloidal divertor system with $4 \mathrm{X}$-points; therefore, one expects 8 strike points in front of the DED coils. At low DED current, the pairs of the strike zone merge together such that one sees only four strike lines. With increasing current the strike zones split more and more similar to the splitting of the strike zones in a poloidal divertor. The similarity between poloidal and helical divertor are very high which even yields for the existence of a private flux zone.

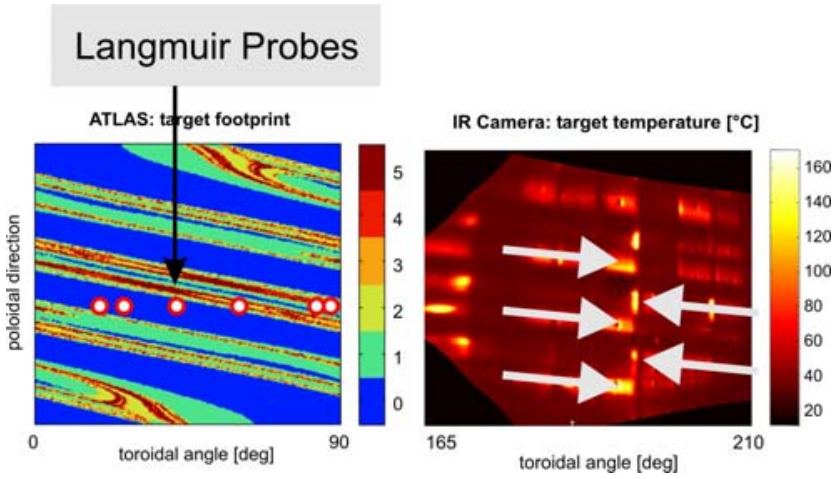

Figure 9: The top of the subfigure shows the power deposition pattern on the divertor target plates during DED operation while the left subfigure shows the result of a $3 D$ plasma transport modeling for the same condition. The arrows in the right subfigure indicate the direction of the incoming flow.

The DED tile alignment shows some imperfections. These imperfections can be utilized to show that the heat flux of neighboring strike zones arrives from opposite direction. The power flux heats either sharply the protruding 
edges of the tiles or - if arriving from the opposite direction - the whole tiles. The arrows show the power flow direction.

Figure 9 shows also the location of Langmuir probes $^{19}$. These probes are utilized to measure the local particle flux, electron temperature and plasma potential in front of the divertor target plate which are displayed in Fig. 10. For the measurements, seven probes are used which are indicated by different colors; in addition, the DED field is swept slowly such that one obtains a nice overlap of the data. In addition to the experimental data, the connection lengths of the magnetic field lines (in units of poloidal turns, right scale) are plotted. The data cover a toroidal angle of $90^{\circ}$ which corresponds to one pair of divertor strike lines.
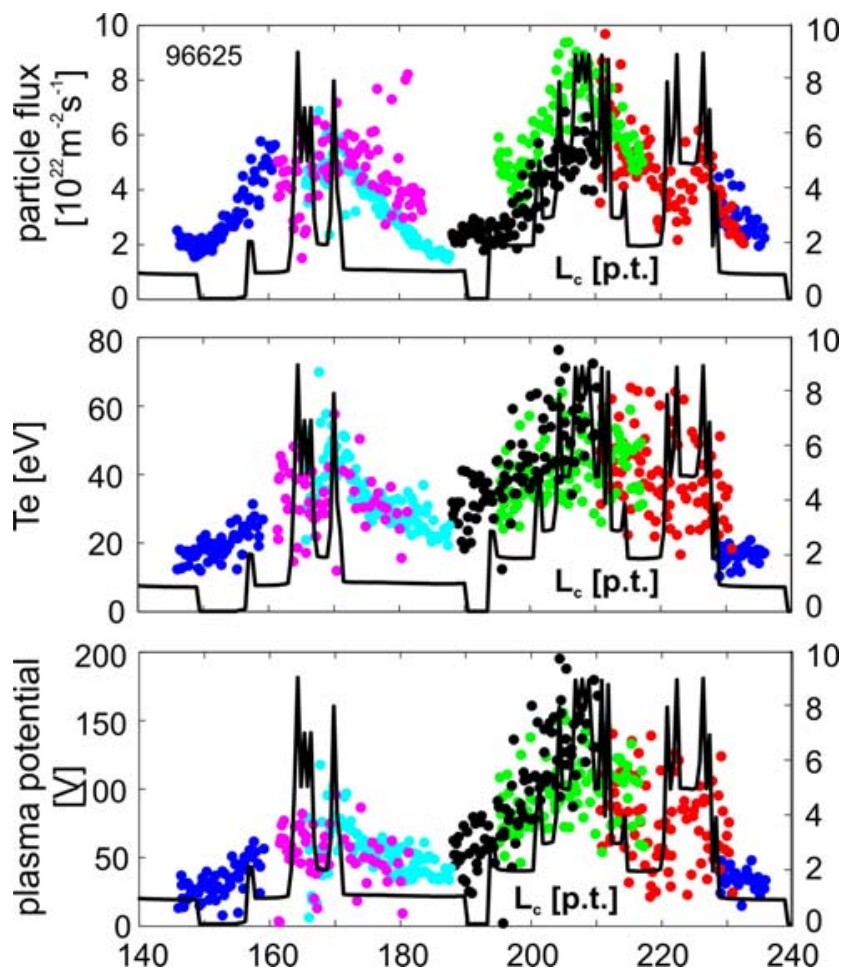

Figure 10: The subfigures shows the particle flux, the electron density and the plasma potential measured by 7 Langmuir probes as a function of the toroidal angle. In addition, the connection length of the magnetic field lines is displayed.

One finds a good correlation between the particle and power fluxes and the structure of the fingers. However, in agreement with our earlier modeling, the particles are not restricted to the location of the fingers, which connect the ergodic zone with the target plate; particles diffuse beyond this layer and fill the laminar zone. Also in agreement with the expectations is the asymmetry of the pair of divertor strike lines. In contrast to the poloidal divertor, the toroidal symmetry is lost and the details of the fingers reaching from the ergodic zone to the divertor are highly complicated.

\section{THE DYNAMIC ASPECT}

Unfortunately, because of the limited space we can give here only a very limited overview about results obtained by the DED. In particular we have only discussed divertor properties of the helical divertor in the $m / n=12 / 4$ base mode. We have neglected the other mode of operation, the $m / n=3 / 1$ base mode; even though some divertor properties are clearer in this configuration, one faces the problem that internal tearing modes are easily excited which make some interpretations more difficult. Another important aspect not touched up to now is the dynamic feature of the DED. Even though this is a characteristic element of the Dynamic Ergodic Divertor we can only make a few remarks here. The motivation for applying different frequency ranges is:

- The application of a low frequency - $50 \mathrm{~Hz}\left(\mathrm{v}_{\mathrm{ph}}=12\right.$ $\mathrm{m} / \mathrm{s}$ ) proposed for technical convenience - is aimed at spreading the heat load evenly either over the protection tiles of the helical divertor located at the high field side or over the pump limiter located at the low field side. Shifting the plasma position and/or changing the plasma aperture allows the control of the relative distribution of the total heat and particle flux between these two main plasma facing components. This puts the proposed DED program in a unique position to answer a number of critical questions which address the physics of mixed ergodic-island layers and how they can be used as an adaptive interface between hot, well-confined plasmas and plasma facing components.

- In contrast to the $50 \mathrm{~Hz}$ case, the medium frequency of $1 \mathrm{kHz}\left(\mathrm{v}_{\mathrm{ph}}=240 \mathrm{~m} / \mathrm{s}\right)$ opens experimental access to the interesting question of whether a rotation of the perturbation pattern which is faster than the transit time of recycling particles - penetrating the boundary layer - will affect particle transport and in consequence also the recycling process and the screening efficiency.

- Last but not least, by applying the upper frequency band of $1 \mathrm{kHz}$ to $10 \mathrm{kHz}$ - the velocity of the perturbation is then of the same order as the natural diamagnetic drift velocity of the plasma - one can investigate whether the rotating field will induce an angular momentum in the plasma and whether the resulting torque ${ }^{20}$ will affect confinement and stability properties.

The experimental results show differences as compared to the initial ideas of the plasma acceleration. It turns out that the torque transfer is not the dominant feature for the plasma acceleration: The DED-field accelerates always the plasma in co-direction of the plasma current, independent whether DC or AC field are applied on the DED 
coils and independent whether the plasma has a large initial velocity or not. The acquired toroidal plasma velocity (difference of velocity relative to the initial velocity) is shown in Fig. 11 for different DED conditions. The velocity gain can be described by a third power parabola as a function of the DED current. The present interpretation of the plasma acceleration is the formation of an edge electric field due to the open field lines where electrons have a higher mobility than ions. The direction of imposed rotation and the observed magnitude of rotation agree with this

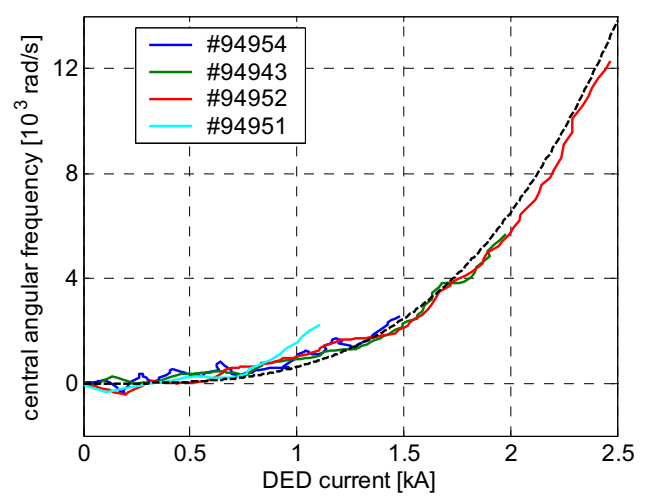

Figure 11: Gain of incremental toroidal plasma rotation as a function of the applied DED current.

picture.

\section{OUTLOOK}

The technical solution for the DED on TEXTOR has been chosen to provide a wide range of experimental possibilities with a limited investment, the aim being the exploration of the potential and the limits of the DED as a means to influence and control plasma-wall interaction. The benefit of the intended studies is seen in the improvement of the understanding of edge transport, island formation, effects of ergodicity etc. which may lead to the incorporation of some features of the TEXTOR DED into other exhaust concepts and may also stimulate the search for novel concepts and technical solutions which would have potential for development towards ultimate application on a burning fusion plasma. Such a very recent development is the suppression of ELMs in an H-mode plasma.

\section{REFERENCES}

1. T. E. Evans, R. A. Moyer, P. R. Thomas et al., "Suppression of Large Edge-Localized Modes in HighConfinement DIII-D Plasmas", Phys. Rev. Lett., 92 (2004) 235003
2. K.H. Finken, S.S. Abdullaev, M.F.M. de Bock et al., "Toroidal plasma rotation induced by the Dynamic Ergodic Divertor in the TEXTOR tokamak", Phys. Rev. Lett., 94 (2005)015003-1

3. K H Finken, S S Abdullaev, W Biel et al., "The dynamic ergodic divertor in the TEXTOR tokamak: plasma response to dynamic helical magnetic field perturbations", Plasma Phys. Control. Fusion 46 (2004) B143-B155

4. Liang Y, Koslowski HR, Kelly, F.A., et al., "Influence of the Dynamic Ergodic Divertor on the Density Limit in TEXTOR", Phys Rev Lett. 94 (2005) 105003

5. S.S. Abdullaev, K.H. Finken, K.H. Spatschek, ,Asymptotical and Mapping Methods in Study of Ergodic Divertor Magnetic Field in a Toroidal System“", Physics of Plasmas, 6 (1999) 153

6. K.H. Finken, S.S. Abdullaev, A. Kaleck, G.H. Wolf, „Operating Space of the Dynamic Ergodic Divertor for TEXTOR-94“" Nucl. Fusion, 39 (1999)637

7. J.A. Lichtenberg, M.A. Liebermann, ,Regular and Stochastic Motion", Applied Mathematical Sciences 38, Springer Verlag, New York, 1983

8. M.C. Gutzwiller, „Chaos in Classics and Quantum Mechanics“, Interdisciplinary Applied Mathematics, Springer Verlag, New York, 1990

9. W. Horton, Y-H Ichikawa, „Chaos and Structures in Nonlinear Plasmas“, World Scientific, Singapore, 1996

10. R. J. Goldston, P.H. Rutherford, „Introduction to Plasma Physics", in IOP Publishing Ltd, 1995

11. B.V. Chirikov, Physics Reports, „A Universal Instability of Many-Dimensional Oscillator Systems" Physics Reports, 52 265,(1979)

12. Jakubowski, M. W.; Abdullaev, S. S.; Finken, K. H., "The atlas and the thermography for TEXTOR-DED operations", Nuclear Fusion, 44 (2004), S. 1 - 11

13. K.H. Finken, T. Eich, A. Kaleck, „First Modelling of the TEXTOR DED Near Field Divertor", Nucl. Fusion, 38 (1998) 515

14. M. Kobayashi, "Modelling approach to 3D simulation of transport in the TEXTOR-DED Laminar Zone with a Finite Element method", J. Nucl. Mater., 313 (2003) 1056

15. A. Samain, T. Blenski, Ph. Ghendrih et al., „Theory of the Ergodic Divertor" Contrib. to Plasma Physics, 30 (1990) 157

16. Ph. Ghendrih, H. Capes, J. Nguyen, A. Samain, „Control of the Edge Transport with the Ergodic Divertor" Contrib. to Plasma Physics, 32 (1992) 179

17. C. de Michelis, A. Grosman, X. Garbet et al., „Characteristics of Ergodic Divertor Plasmas in the TORE SUPRA Tokamak" Nucl. Fusion, 35, 1133,(1995)

18. M.W. Jakubowski, S.S. Abdullaev, K.H. Finken, M. Lehnen and TEXTOR team, "Heat deposition patterns on the target plates of the dynamic ergodic divertor", J. Nucl. Mater., 337-339 (2005) 176-180

19. Lehnen, M., Finken, K.H., Jakubowski,et al., "First results from the dynamic ergodic divertor at TEXTOR", Journal of Nuclear Materials, 337-339 (2005) 171-175

20. K.H. Finken, „Perturbation Field Penetration into the TEXTOR Tokamak and the Resulting Torque“, Nucl. Fusion, 39 (1999)707 